\title{
Médiévales
}

Langues, Textes, Histoire

66 | printemps 2014

Harmonie Disharmonie

\section{Thomas M. CHARLES-EDWARDS, Wales and the Britons.} 350-1064

Oxford, Oxford University Press, 2013 (« The History of Wales », vol. 1), $\mathrm{xx}-796 \mathrm{p}$.

\section{Alban Gautier}

\section{(2) OpenEdition}

Journals

Édition électronique

URL : https://journals.openedition.org/medievales/7308

DOI : $10.4000 /$ medievales. 7308

ISSN : $1777-5892$

Éditeur

Presses universitaires de Vincennes

Édition imprimée

Date de publication : 30 juin 2014

Pagination : 215-216

ISBN : 978-2-84292-405-8

ISSN : 0751-2708

Référence électronique

Alban Gautier, «Thomas M. charles-edwards, Wales and the Britons. 350-1064 », Médiévales [En ligne], 66 | printemps 2014, mis en ligne le 10 juillet 2014, consulté le 22 avril 2022. URL : http:// journals.openedition.org/medievales/7308; DOI : https://doi.org/10.4000/medievales.7308

Ce document a été généré automatiquement le 22 avril 2022

Tous droits réservés 


\section{Thomas M. CHARLES-EDWARDS, Wales and the Britons. 350-1064}

Oxford, Oxford University Press, 2013 (« The History of Wales », vol. 1), $\mathrm{xx}-796 \mathrm{p}$.

\section{Alban Gautier}

\section{RÉFÉRENCE}

Thomas M. CHARLES-EDWARDS, Wales and the Britons. 350-1064, Oxford, Oxford University

Press (« The History of Wales », vol. 1), 2013, Xx - 796 p.

1 Treize ans après une magistrale histoire de l'Irlande dans les premiers siècles médiévaux $^{1}, T$. M. Charles-Edwards réédite l'exploit d'écrire une histoire en un volume, complète et même fouillée, et se penche cette fois-ci sur un autre espace: ce livre constitue en effet le premier volume d'une histoire générale du pays de Galles, projet au très long cours dont quatre autres volumes sont parus depuis 1980 et dont un cinquième (sur le XIX ${ }^{\mathrm{e}}$ siècle) est encore annoncé. L'attente a donc été longue, mais elle valait la peine: on peut dire sans trop s'avancer que cet ouvrage est une somme qui dominera pendant plusieurs décennies l'étude de l'histoire galloise et des mondes brittoniques. Aussi, plutôt que de faire la liste des qualités de ce livre ou d'en donner les résumés (choses tout à fait impossibles), nous partirons dans les lignes qui suivent de ce qu'il n'est pas : le lecteur non averti risque en effet d'être dérouté par certaines de ses caractéristiques, et une mise en garde n'a rien d'inutile.

2 D'abord, ce livre n'est pas une introduction à l'histoire du pays de Galles dans le haut Moyen Âge. À cet égard, il ne remplace ni ne dépasse le livre ancien mais toujours important de W. Davies, plus court, plus maniable et sans doute plus clair ${ }^{2}$. C'est ainsi que la plupart de la trentaine de cartes que compte le livre de T. M. Charles-Edwards ne sont pas des cartes de synthèse, proposant une cartographie de la géographie politique ou religieuse du pays de Galles au haut Moyen Âge : ce sont pour l'essentiel des cartes visant à illustrer le propos, soit en fournissant des localisations, soit en donnant à voir 
un point argumenté dans le texte. De même, la rareté des propos récapitulatifs et l'absence de conclusion générale montrent que le projet de l'auteur n'est pas de dégager les grandes lignes de force de la période. Ce livre ne propose donc pas de synthèses consensuelles sur les grands sujets discutés et disputés de l'histoire du pays de Galles: l'auteur expose soigneusement les différentes positions, mais prend luimême parti, parfois de façon très nette. C'est le cas, pour ne donner qu'un exemple, de la question d'une latinité insulaire - en d'autres termes, de l'existence ou non d'un latin vulgaire puis d'une langue romane dans l'île de Bretagne pendant l'époque romaine et après. Pour l'auteur, non seulement cette langue a existé, mais elle a survécu jusqu'au $\mathrm{VII}^{\mathrm{e}}$ siècle, au moins dans certaines régions et certains milieux; et il fournit au lecteur une batterie d'arguments linguistiques et épigraphiques allant dans ce sens.

Il n'est donc a priori pas question pour le lecteur de lire ce livre de la première à la dernière page. Ce livre est avant tout un précis d'histoire galloise et brittonique du haut Moyen Âge : l'auteur y traite divers dossiers, dont plusieurs se répondent d'un passage à un autre, mais qui ne dessinent l'histoire de la région que comme une sorte de patchwork. Cette impression est confirmée par la longue bibliographie de soixante pages où les ouvrages sont classés par thèmes, et surtout par l'incroyable index de cinquante-quatre pages, extrêmement détaillé : ce livre n'est pas d'abord fait pour être lu, mais pour être consulté. C'est de fait une mine extraordinaire de renseignements et d'analyses sur les sources de toutes sortes (poésie, lois, épigraphie, histoire, hagiographie, etc.). L'archéologie aurait sans doute pu être un peu plus présente, mais elle n'est jamais entièrement oubliée. L'image de ce haut Moyen Âge brittonique est donc dessinée à travers des études précises qui se succèdent et que l'auteur traite toujours de manière exhaustive. Parmi une bonne centaine de dossiers, on mentionnera un peu au hasard, et afin de montrer la variété des sujets abordés, le sens des termes uncia et modius dans les chartes, l'essor de la dynastie des Merfynion au IX siècle, le fonctionnement de la métrique galloise, la nature exacte des églises appelées clas, ou encore les différentes analyses proposées pour l'ouvrage de terrassement appelé Offa's Dyke (le fossé d'offa).

Contrairement à ce que le titre de la collection pourrait laisser croire, mais conformément à ce qu'annonce le titre du livre, le lecteur n'a pas non plus sous les yeux une simple histoire du pays de Galles, limitée aux seules régions définies comme telles au cours du bas Moyen Âge. Il s'agit d'une histoire «du pays de Galles et des Bretons » entre le dernier siècle de la présence romaine dans l'île et la mort en 1064 de Gruffudd ap Llewelyn, seul souverain à avoir réuni pour un temps sous son autorité l'ensemble des territoires qui formeraient le pays de Galles. L'auteur ne cesse donc de traverser les frontières du pays de Galles pour envisager l'ensemble des peuples brittoniques, surtout (mais pas seulement) insulaires: Gallois au sens étroit, mais aussi Cornouaillais, habitants des régions aujourd'hui situées à la frontière de l'Angleterre et de l'Écosse (royaume des Gododdin, Strathclyde, Cumbria), Bretons vivant parmi les Anglo-Saxons et peu à peu assimilés à cette nouvelle culture, mais aussi Bretons d'Armorique. Ces populations sont, dans de nombreux cas, étudiées ensemble, à la fois pour des raisons pratiques (il n'est pas toujours possible de s'assurer de la provenance de tel ou tel manuscrit, ou de distinguer un poème du pays de Galles d'un poème d'origine cumbrien) et pour des raisons historiques: le lent passage, dont l'auteur montre bien qu'il n'a rien de linéaire, d'une identité "bretonne " partagée à des identités « cornouaillaise » ou « galloise » distinctes est précisément un des rares traits 
de la période dont l'auteur cherche à dégager la logique sur le long terme. Par ailleurs, ces «Bretons » sont toujours étudiés dans leurs rapports avec leurs voisins et avec tous les peuples qui sont en contact avec eux: avant tout les Anglo-Saxons, que ceux-ci soient Northumbriens, Merciens ou Ouest-Saxons, mais aussi les Romains, les Irlandais, les Pictes, les Francs ou les Scandinaves. Il s'agit donc d'une histoire des Bretons et des Gallois, mais sans cesse replacés dans un environnement historique, culturel ou religieux plus vaste.

\section{NOTES}

1. T. M. CHARLES-EDWARDS, Early Christian Ireland, Oxford, 2000.

2. W. DAVIES, Wales in the Early Middle Ages, Leicester, 1982. 